\title{
Utilização Off-label dos Stents Farmacológicos nas Intervenções Coronárias Percutâneas: Registro da Clínica São Vicente
}

\author{
José Ary Boechat e Salles ${ }^{1,2,3}$, Júlio César Machado Andréa ${ }^{1,2}$, Leandro Assumpção Cortes², \\ Filipe Goldberg' ${ }^{2}$, Lilian Carestiato', Felipe Camillis ${ }^{1}$, Hélio Roque Figueira ${ }^{1,2,3}$
}

\section{RESUMO}

Fundamentos: Estudos clínicos randomizados indicam que os stents farmacológicos reduzem a taxa de reestenose e a necessidade de procedimentos de revascularização. Objetivo: Avaliar o desempenho dos stents farmacológicos no mundo-real, comparando a evolução dos pacientes elegíveis nos estudos randomizados (on-label) com aqueles inelegíveis para intervenção coronária percutânea com stents farmacológicos (off-label). Método: Entre junho de 2002 e dezembro de 2007, 653 pacientes foram tratados por intervenção coronária percutânea somente com stents farmacológicos: 309 pacientes com stents farmacológicos em indicações on-label (309 stents) e 344 pacientes com presença de ao menos uma indicação offlabel (654 stents). Foram consideradas indicações offlabel: fração de ejeção < 25\%, múltiplos stents, oclusão crônica, tronco não protegido, bifurcação, ponte de safena, infarto agudo, lesão ostial e lesão reestenótica. Avaliamos a evolução hospitalar e no período médio de 23,2 meses após o procedimento. Resultados: Sucesso angiográfico elevado, com maior ocorrência de no-reflow $(0 \%$ vs. $1,5 \% ; p=0,04)$ e mionecrose $(1,9 \%$ vs. $5,2 \% ; p=0,02)$, foi observado nos pacientes off-label, porém sem diferença na mortalidade $(0,3 \%$ vs. $0,9 \% ; p=0,3)$. No seguimento tardio de $80 \%$ dos pacientes por 23,2 meses, foram observadas reduzida incidência de eventos cardíacos maiores $(8 \%$ vs. $8,1 \% ; p=0,5)$ e de revascularização da lesão-alvo $(6,8 \%$ vs. $5,1 \%$; $p=0,2)$. Conclusão: $O$ implante off-label do stent farmacológico é seguro, com baixa mortalidade. Apesar da maior complexidade, não verificamos aumento do risco de eventos cardíacos adversos tardios ou revascularização da lesão-alvo quando comparado com o implante on-label.

DESCRITORES: Stents farmacológicos. Contenedores. Angioplastia transluminal percutânea coronária. Avaliação de resultados.

1 Clínica São Vicente - Rio de Janeiro, RJ.

2 Hospital Cardiotrauma - Rio de Janeiro, RJ.

3 Hospital de Clínicas de Niterói - Rio de Janeiro, RJ.

Correspondência: José Ary Boechat e Salles. Rua João Borges, 204

- Gávea - Rio de Janeiro, RJ - CEP 22451-100

E-mail: josearyboechat@gmail.com

Recebido em: 29/7/2008 • Aceito em: 17/9/2008

\section{SUMMARY}

Off-Label Utilization of Drug-Eluting Stents in Percutaneous Coronary Intervention: Clínica São Vicente Registry

Background: Randomized clinical trials indicate that drugeluting stents (DES) reduce the rates of restenosis and need for subsequent revascularization procedures. Objective: This study examined the performance of DES in a 'real world' setting comparing the outcomes of trial-eligible (on-label) versus ineligible (off-label) patients undergoing percutaneous coronary interventions. Methods: From June 2002 to December 2007, 653 patients underwent percutaneous coronary intervention exclusively using DES: 309 patients (309 stents) with on-label indications, and 344 patients (654 stents) with at least one off-label indication. Off-label indications were considered ejection fraction $<25 \%$, multiple DES, chronic total occlusions, unprotected left main coronary artery, bifurcations, saphenous vein grafts, acute myocardial infarction, ostial or restenotic lesions. We evaluated in-hospital as well as late follow-up events. Results: A high angiographic success, but with higher in-hospital myonecrosis $(1.9 \%$ vs $5.2 \% ; \mathrm{p}=0.02)$ and noreflow phenomenon $(0 \%$ vs $1.5 \%$; $p=0.04)$, was observed with off-label use; the incidence of death $(0.3 \%$ vs $0.9 \%$; $p=0.3)$ was not statistically different between groups. Data obtained from $80 \%$ of the patients over an average 23.2-month follow-up period showed a low incidence of major adverse cardiac events ( $8 \%$ vs. $8.1 \%$; $p=0.5)$ and target vessel revascularization procedures $(6.8 \%$ vs. $5.1 \% ; p=0.2$. Conclusion: Off-label implantation of DES is safe, with a low mortality rate. DES use in this complex cohort of patients does not confer a higher risk of late adverse cardiac events or repeat revascularizations when compared to on-label indications.

DESCRIPTORS: Drug eluting stents. Stents. Angioplasty, transluminal, percutaneous, coronary. Outcome assessment.

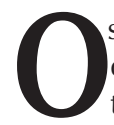
stents farmacológicos reduziram a ocorrência de reestenoses angiográfica e clínica nos pacientes tratados por intervenção coronária percutânea, quando comparados com stents convencionais. No 
entanto, os tipos de lesões selecionadas para estudo nos ensaios randomizados não refletem inteiramente o largo espectro da doença coronária que é tratada rotineiramente nos laboratórios de Hemodinâmica. A utilização de stents farmacológicos pelos intervencionistas expandiu-se, com o uso dos stents em indicações não especificadas pelos fabricantes (indicações off-label).

\section{MÉTODO}

\section{População estudada}

Estudo retrospectivo de uma série consecutiva de pacientes tratados por intervenção coronária percutânea exclusivamente com implante de stents farmacológicos, realizado em três instituições (Clínica São Vicente, Hospital Cardiotrauma e Hospital de Clínicas de Niterói), localizadas no Rio de Janeiro. Entre junho de 2002 e dezembro de 2007, foram catalogadas 2.369 angioplastias no banco de dados de nossa instituição, sendo 653 com implante exclusivo de stents farmacológicos. A essa coorte de casos deu-se o nome de Registro da Clínica São Vicente. Para o estudo em questão, foram excluídos os pacientes com implante simultâneo de stents farmacológico e convencional.

Segundo os critérios utilizados em estudos randomizados iniciais com stents farmacológicos, foram definidos como on-label os portadores de doença coronária sintomática decorrente de lesão de novo em vasos nativos, com estenoses com comprimento inferior a $30 \mathrm{~mm}$ em vasos de diâmetro de 2,5 $\mathrm{mm}$ a 3,5 $\mathrm{mm}$, e como pacientes off-label aqueles com ao menos uma das indicações a seguir: fração de ejeção < 25\%, múltiplos stents, oclusão crônica, tronco não protegido, bifurcação, ponte de safena, infarto agudo, lesão ostial e tratamento de lesão reestenótica.

\section{Procedimento de intervenção}

Os pacientes receberam terapia antiplaquetária dupla pré-intervenção, com aspirina $200 \mathrm{mg} /$ dia e clopidogrel $75 \mathrm{mg} / \mathrm{dia}$ ou ticlopidina $250 \mathrm{mg} / \mathrm{dia}$, iniciada pelo menos três dias antes nos casos eletivos. Pacientes com síndromes coronárias agudas receberam dose de ataque de $600 \mathrm{mg}$ de clopidogrel pelo menos duas a seis horas antes do procedimento, com uso, em casos selecionados e a critério do operador, de inibidores intravenosos da glicoproteína IIb/IIla (casos de trombo angiográfico e infarto agudo do miocárdio).

O procedimento intervencionista padrão foi realizado por punção arterial femoral ou radial, tendo sido realizada pré-dilatação a critério do operador, seguida do implante de stent adequado ao tamanho do vaso e ao comprimento da lesão por análise visual. Impactação pós-implante foi realizada, preferencialmente, com balão não complacente acima de $12 \mathrm{~atm}$. O uso de ultrasom intracoronário foi determinado pelo operador, com vistas ao implante ótimo do stent (segundo crité- rios definidos no estudo MUSIC). Após o procedimento, todos os pacientes receberam orientação para uso de aspirina $200 \mathrm{mg} /$ dia indefinidamente e clopidogrel $75 \mathrm{mg} / \mathrm{dia}$ ou ticlopidina $250 \mathrm{mg} / \mathrm{dia}$ por seis a doze meses. Acompanhamento clínico foi realizado pósprocedimento, com eletrocardiograma, dosagem enzimática (CK-total, fração MB e troponina), hematócrito e creatinina sérica.

\section{Definição das variáveis intra-hospitalares}

As variáveis intra-hospitalares foram definidas da seguinte forma: sucesso angiográfico, como lesão residual intra-stent inferior a $10 \%$ e fluxo distal normal; sucesso clínico, como sucesso angiográfico na ausência de infarto, fenômeno de no-reflow, trombose de stent e óbito; infarto pós-procedimento, como elevação do segmento ST no eletrocardiograma evolutivo, desenvolvimento de nova onda $\mathrm{Q}$ em duas derivações eletrocardiográficas contíguas ou novo bloqueio de ramo esquerdo e/ou evidência bioquímica de infarto do miocárdio com elevação da creatina quinase acima de duas vezes o valor normal, associada a aumento da fração $M B$ três vezes acima do limite da normalidade e/ou elevação sérica da troponina acima do valor de $0,5 \mathrm{ng} / \mathrm{ml}$; trombose aguda de stent, como documentação angiográfica de trombo intra-stent, com fluxo distal TIMI 0 ou 1 nas primeiras 24 horas pósprocedimento; e trombose subaguda de stent, como documentação angiográfica de trombo intra-stent, com fluxo distal TIMI 0 ou 1 entre 24 horas e 30 dias pósprocedimento.

Acompanhamento tardio foi feito por meio de seguimento clínico e questionário telefônico anual, com apuração do quadro clínico e verificação da ocorrência de eventos cardíacos maiores (óbito, infarto do miocárdio e novas intervenções).

\section{Definição das variáveis pós-hospitalares}

As variáveis pós-hospitalares foram definidas da seguinte forma: infarto tardio, como reinternação com diagnóstico médico de infarto do miocárdio; revascularização do vaso-alvo, como necessidade de reintervenção para tratamento do vaso previamente tratado por nova angioplastia ou cirurgia de revascularização do miocárdio; e evento cardíaco adverso maior (dados cumulativos), como ocorrência de qualquer desfecho clínico desfavorável, quer seja infarto tardio, nova intervenção percutânea ou cirúrgica, ou óbito.

\section{Análise estatística}

A comparação entre as variáveis quantitativas foi feita com o teste $t$ de Student. As comparações referentes às variáveis qualitativas foram feitas com o teste estatístico do qui-quadrado, utilizando programa SPSS para Windows, versão 15.1 (SPSS Inc., Chicago, IL, Estados Unidos). Consideraram-se significantes variáveis com valor de $p<0,05$. 


\section{RESULTADOS}

No período de junho de 2002 a dezembro de 2007, 2.369 pacientes foram tratados por angioplastia em nosso Serviço, dos quais 762 somente com stents farmacológicos. Após aplicação dos critérios de exclusão previamente descritos, foram selecionados 653 pacientes.

$\mathrm{Na}$ Tabela 1 estão apresentadas as características clínicas dos pacientes, observando-se maior número de homens, de insuficiência renal e de história de intervenção coronária prévia no grupo off-label, caracterizando um segmento de pacientes com doença coronária mais extensa e grave. Na Tabela 2 estão descritas as características angiográficas dos dois grupos, com presença de múltiplos fatores angiográficos desfavoráveis no grupo off-label, com estenoses mais longas e complexas, bem como presença de trombo, angulação acentuada e calcificação, reduzindo a incidência de implante direto de stent e aumentando o uso de múltiplos stents e inibidores de glicoproteína.

A maior complexidade clínica e angiográfica no grupo off-label se traduziu pela maior ocorrência de fenômeno de no-reflow e infarto pós-procedimento, com menor sucesso clínico. No entanto, apesar da maior ocorrência de eventos adversos, não houve diferença na mortalidade intra-hospitalar (Tabela 3).

No seguimento tardio, realizado em $80 \%$ dos pacientes por um período médio de 23,2 meses (Figura 1), foram registrados os eventos cardíacos adversos maiores $(8 \%$ vs. $8,1 \% ; p=0,5)$ e a revascularização do vaso-alvo $(6,8 \%$ vs. $5,1 \% ; p=0,2)$, não tendo sido observada diferença entre os grupos.

\section{DISCUSSÃO}

Os stents farmacológicos reduziram a incidência de reestenoses clínica e angiográfica nos pacientes tratados por intervenção coronária percutânea nos estudos randomizados. Foram inicialmente implantados em populações altamente selecionadas, visando ao tratamento de lesões não complexas ${ }^{1,2}$, com benefício clínico e angiográfico mantido por até quatro anos $^{3}$. A indicação inicial do fabricante (on-label) do stent revestido com sirolimus (Cypher $\left.{ }^{\circledR}\right)$ foi para o implante nas lesões de novo em vasos nativos com comprimento inferior a $30 \mathrm{~mm}$ em vasos de diâmetro de 2,5 $\mathrm{mm}$ a 3,5 $\mathrm{mm}$. A indicação do fabricante do stent revestido com paclitaxel (Taxus ${ }^{\circledR}$ ) diferia somente no comprimento do stent $(<28 \mathrm{~mm})$ e no limite superior do diâmetro do vaso (até $3,75 \mathrm{~mm}$ ).

As lesões selecionadas nos ensaios randomizados, entretanto, não refletem o largo espectro da doença coronária que é tratada rotineiramente nos laboratórios de Hemodinâmica. Assim, a utilização dos stents farmacológicos pelos intervencionistas expandiu-se, com implante em indicações não contidas na "bula" do fabricante, denominadas assim "indicações off-label".

TABELA 1

Características clínicas dos pacientes

\begin{tabular}{|c|c|c|c|}
\hline & On-label & Off-label & $\mathbf{p}$ \\
\hline Pacientes & $n=309$ & $\mathrm{n}=344$ & - \\
\hline Idade $>70$ anos & $82(26,5 \%)$ & $95(27,6 \%)$ & 0,4 \\
\hline Masculino & $190(61,5)$ & $256(74,4)$ & $<0,001$ \\
\hline \multicolumn{4}{|l|}{ Fatores de risco } \\
\hline Hipertensão & $227(73,5 \%)$ & $259(75,3 \%)$ & 0,3 \\
\hline Hipercolesterolemia & $215(69,6 \%)$ & $212(64,2 \%)$ & 0,08 \\
\hline Diabetes melito & $118(38,2 \%)$ & $119(34,6 \%)$ & 0,1 \\
\hline Insuficiência renal & $12(3,9 \%)$ & $30(8,7 \%)$ & 0,008 \\
\hline Tabagismo & $41(13,3 \%)$ & $47(13,7 \%)$ & 0,4 \\
\hline \multicolumn{4}{|l|}{ Quadro clínico } \\
\hline Angina estável & $144(46,6 \%)$ & $161(46,8 \%)$ & 0,4 \\
\hline Angina instável & $125(40,5 \%)$ & $116(33,7 \%)$ & 0,1 \\
\hline Infarto sem supra ST & $40(12,9 \%)$ & $54(15,7 \%)$ & 0,1 \\
\hline Infarto com supra ST & 0 & $13(3,8 \%)$ & $<0,001$ \\
\hline \multicolumn{4}{|l|}{ Histórico } \\
\hline CRM prévia & $36(11,7 \%)$ & $82(23,8 \%)$ & $<0,001$ \\
\hline ATC prévia & $12(3,9 \%)$ & $11(3,2 \%)$ & 0,3 \\
\hline Infarto prévio & $55(17,8 \%)$ & $99(28,8 \%)$ & 0,001 \\
\hline
\end{tabular}

ATC = angioplastia transluminal coronária; CRM = cirurgia de revascularização do miocárdio; $\mathrm{n}$ = número de pacientes. 
TABELA 2

Características angiográficas e do procedimento

\begin{tabular}{|c|c|c|c|}
\hline & On-label $(\mathrm{n}=309)$ & Off-label $(\mathrm{n}=344)$ & $\mathbf{p}$ \\
\hline \multicolumn{4}{|l|}{ Gravidade da doença coronária } \\
\hline Multiarterial & $153(49,5 \%)$ & 248 (72,1\%) & $<0,001$ \\
\hline Múltiplos stents farmacológicos & 0 & $216(62,6 \%)$ & $<0,001$ \\
\hline Disfunção grave do VE & 0 & $24(7 \%)$ & $<0,001$ \\
\hline \multicolumn{4}{|l|}{ Indicação de stent } \\
\hline Lesão de novo & $309(100 \%)$ & $266(77,3 \%)$ & $<0,001$ \\
\hline Lesão reestenótica & 0 & $78(22,7 \%)$ & $<0,001$ \\
\hline \multicolumn{4}{|l|}{ Características das lesões } \\
\hline Ostiais & 0 & $55(16 \%)$ & $<0,001$ \\
\hline Trombo & $14(4,5 \%)$ & $38(11 \%)$ & 0,001 \\
\hline Lesão > 10 mm & $225(72,8 \%)$ & $272(79,1 \%)$ & 0,03 \\
\hline Angulação < 90 graus & $11(3,6 \%)$ & $25(7,3 \%)$ & 0,02 \\
\hline Calcificadas & $73(23,6 \%)$ & $102(29,7 \%)$ & 0,05 \\
\hline Ulceradas & $118(38,2 \%)$ & $170(49,4 \%)$ & 0,002 \\
\hline Excêntricas & $278(90 \%)$ & $296(86 \%)$ & 0,07 \\
\hline Oclusão crônica & 0 & $12(3,5 \%)$ & $<0,001$ \\
\hline Bifurcação & 0 & $64(18,6 \%)$ & $<0,001$ \\
\hline Stent $<3 \mathrm{~mm}$ & $111(35,9 \%)$ & $142(41,3 \%)$ & 0,09 \\
\hline Stent $>20 \mathrm{~mm}$ & $129(41,7 \%)$ & $216(62,8 \%)$ & $<0,001$ \\
\hline Lesão tipo B2/C & $171(55,3 \%)$ & $280(81,4 \%)$ & $<0,001$ \\
\hline \multicolumn{4}{|l|}{ Vaso abordado } \\
\hline Tronco não protegido & 0 & $3(0,9 \%)$ & 0,1 \\
\hline Descendente anterior & $203(65,7 \%)$ & $199(57,8 \%)$ & 0,02 \\
\hline Circunflexa & $55(17,8 \%)$ & $92(26,7 \%)$ & 0,004 \\
\hline Coronária direita & $55(17,8 \%)$ & $101(29,4 \%)$ & $<0,001$ \\
\hline Ponte de safena & 0 & $26(7,6 \%)$ & $<0,001$ \\
\hline \multicolumn{4}{|l|}{ Dados do procedimento } \\
\hline Stent direto & $193(62,5 \%)$ & $183(53,2 \%)$ & 0,01 \\
\hline Ultra-som intracoronário & $137(44,3 \%)$ & $152(44,2 \%)$ & 0,5 \\
\hline Inibidor de glicoproteína IIb/IIla & $11(3,6 \%)$ & $22(6,4 \%)$ & 0,06 \\
\hline \multicolumn{4}{|l|}{ Tipo de stent } \\
\hline Taxus $^{\circledR}$ & $185(59,9 \%)$ & $191(55,7 \%)$ & 0,3 \\
\hline Cypher $^{\circledR}$ & $113(36,6 \%)$ & $149(43,4 \%)$ & 0,07 \\
\hline Endeavor $^{\circledR}$ & $6(1,9 \%)$ & $12(3,5 \%)$ & 0,2 \\
\hline Xience $V^{\circledR}$ & $5(1,6 \%)$ & $8(2,3 \%)$ & 0,5 \\
\hline
\end{tabular}

TABELA 3

Evolução intra-hospitalar

\begin{tabular}{lccc}
\hline & On-label $(\mathbf{n}=\mathbf{3 0 9})$ & Off-label $(\mathbf{n}=\mathbf{3 4 4})$ & $\mathbf{p}$ \\
\hline Sucesso angiográfico & $307(99,4 \%)$ & $344(100 \%)$ & 0,2 \\
Sucesso clínico & $300(97,1 \%)$ & $318(92,7 \%)$ & 0,009 \\
No-reflow & 0 & $5(1,5 \%)$ & 0,04 \\
Infarto & $6(1,9 \%)$ & $18(5,2 \%)$ & 0,02 \\
Trombose aguda/subaguda & 0 & 0 & - \\
Óbito & $1(0,3 \%)$ & $3(0,9 \%)$ & 0,3 \\
Cirurgia de emergência & $1(0,3 \%)$ & 0 & 0,4 \\
\hline
\end{tabular}

$\mathrm{n}=$ número de pacientes. 


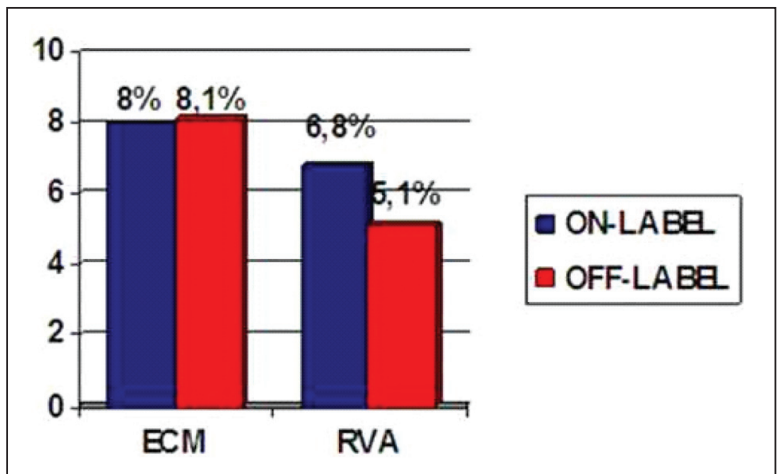

Figura 1 - Incidência de eventos cardíacos adversos maiores e revascularização de vaso-alvo nos grupos com indicação on e offlabel. $\mathrm{ECM}=$ eventos cardíacos adversos maiores; RVA = revascularização do vaso-alvo.

A generalização do uso dos stents farmacológicos foi então o passo seguinte, com uso indiscriminado do stent farmacológico.

No entanto, dados preliminares e controversos de alguns estudos sugerem que o stent farmacológico pode estar associado a pior evolução clínica, principalmente pelo aumento do risco de trombose do stent, com aumento da mortalidade e do infarto do miocárdio, quando comparado ao stent não-farmacológico ${ }^{4-6}$, particularmente em pacientes que não estão recebendo clopidogrel $^{7}$. Em 2006, por causa da preocupação com eventos adversos tardios com o uso de stents farmacológicos, o Food and Drug Administration (FDA) convocou um painel para rever os dados dos estudos randomizados e dos registros ${ }^{8,9}$. Com base na revisão desses dados, esse painel concluiu que o risco de trombose dos stents farmacológicos, quando utilizados nas indicações on-label, é semelhante ao dos stents não-farmacológicos ${ }^{10}$. Em contraste, o painel concluiu que os eventos tardios ocorrem com incidência crescente nas indicações off-label, suscitando preocupação quanto a sua segurança ${ }^{10}$.

O uso off-label ocorre em aproximadamente $60 \%$ das angioplastias com implante de stents farmacológicos. Esses pacientes compõem uma população de maior risco, com numerosas co-morbidades, com lesões com morfologia desfavorável e apresentações clínicas instáveis.

Os resultados hospitalares e tardios com stents farmacológicos em situações off-label são controversos. Apesar dos inúmeros estudos disponíveis sobre stents farmacológicos nos últimos anos, não há registro, até o momento, de algum estudo randomizado comparando a evolução dos pacientes tratados com stents farmacológicos em situações on-label vs. offlabel. Beohar et al. ${ }^{11}$ descreveram um registro multicêntrico com 5.541 pacientes tratados com stents farmacológicos, sendo $47 \%$ deles com indicações offlabel ou não testadas. O risco de morte, infarto do miocárdio ou trombose de stent em trinta dias, bem como a taxa de revascularização do vaso-alvo em um ano, foram significativamente maiores quando os stents farmacológicos foram utilizados em situações off-label, comparativamente às indicações aprovadas. Entretanto, as taxas foram baixas nos dois grupos. Win et al. ${ }^{12}$, em um registro multicêntrico de 3.323 pacientes tratados com stents farmacológicos, dos quais 55\% apresentavam ao menos uma indicação off-label, relataram ter observado letalidade semelhante, porém com aumento da ocorrência de infarto, trombose de stent e revascularização do vaso-alvo, em situações off-label, comparativamente às indicações aprovadas pelo FDA para stent farmacológico. Assim, concluíram que o risco associado ao uso off-label do stent farmacológico não está limitado ao período periprocedimento, mas continua no período após a alta hospitalar. A maior de todas as séries foi relatada por Rao et al. ${ }^{13}$, com 408.033 procedimentos utilizando stents farmacológicos, a partir de dados do National Cardiovascular Data Registry. Quando os stents farmacológicos foram utilizados em indicações off-label (limitadas a infarto agudo, reestenose intra-stent, pontes de safena e oclusão crônica, compreendendo $24,1 \%$ do total), as taxas de eventos intrahospitalares foram inferiores às esperadas pelo modelo validado. Nesse estudo, entretanto, não houve rigor na dosagem enzimática após os procedimentos, além de conter menos critérios off-label, sugerindo que a seleção dos casos e a decisão clínica do uso de stents farmacológicos em indicações off-label podem ser seguras e apropriadas.

Os achados com stents convencionais nas lesões off-label são, em contrapartida, inferiores, como comprovado por Marroquin et al. ${ }^{14}$, em que os pacientes tratados com stents farmacológicos demonstraram melhor evolução, com redução da necessidade de reintervenção, sem aumento da mortalidade e do infarto.

Nesta série, compreendendo uma população de mundo-real, notamos a expansão do uso do stent farmacológico, com implante off-label em mais de 50\% dos casos. Verificamos maior gravidade, extensão e complexidade da doença coronária nesse grupo tradicionalmente associado a resultados desanimadores com stents convencionais, especialmente em lesões ostiais, em bifurcações e em lesões reestenóticas.

Apesar da maior gravidade da população descrita, encontramos resultados hospitalares favoráveis, com reduzida mortalidade, apesar da maior incidência de infarto periprocedimento, atingindo mesmo assim níveis reduzidos e aceitáveis em uma população tão complexa. Os achados no seguimento tardio foram animadores, com evidência de reduzida ocorrência de eventos cardíacos nos dois grupos, demonstrando a manutenção dos resultados intra-hospitalares. Nossa análise apresenta limitações, especialmente por se tratar de uma série não-multicêntrica, com perda de acompanhamento de aproximadamente $20 \%$ dos casos. Entretanto, os dados 
descritos, associados aos da literatura, demonstram que, na prática clínica corrente, o uso dos stents farmacológicos em situações off-label nos parece seguro e eficaz a curto e médio prazos, com necessidade de mais registros, especialmente em nosso País, para esclarecimento de dúvidas principalmente quanto à ocorrência de trombose tardia dos dispositivos.

\section{CONCLUSÃO}

O implante off-label do stent farmacológico é seguro, com baixa mortalidade. Apesar de sua maior complexidade, não verificamos aumento do risco de eventos cardíacos adversos tardios ou revascularização da lesãoalvo quando comparado com o implante on-label.

\section{REFERÊNCIAS BIBLIOGRÁFICAS}

1. Moses JW, Leon MB, Popma JJ, Fitzgerald PJ, Holmes DR, O'Shaughnessy C, et al. Sirolimus-eluting stents versus standard stents in patients with stenosis in a native coronary artery. N Engl J Med. 2003;349(14):1315-23.

2. Stone GW, Ellis SG, Cox DA, Hermiller J, O'Shaughnessy C, Mann JT, et al. One-year clinical results with the slowrelease, polymer-based, paclitaxel-eluting Taxus stent: the TAXUS-IV trial. Circulation. 2004;109(16):1942-7.

3. Sousa JE, Costa MA, Abizaid A, Feres F, Seixas AC, Tanajura $L F$, et al. Four-year angiographic and intravascular ultrasound follow-up of patients treated with sirolimus eluting stents. Circulation. 2005;111(18):2326-9.

4. Pfisterer M, Brunner-La Rocca HP, Buser PT, Rickenbacher P, Hunziker P, Mueller C, et al. Late clinical events after clopidogrel discontinuation may limit the benefit of drugeluting stents: an observational study of drug-eluting versus bare-metal stents. J Am Coll Cardiol. 2006;48(12):2584-91.
5. Bavry AA, Kumbhani DJ, Helton TJ, Borek PP, Mood GR, Bhatt DL. Late thrombosis of drug-eluting stents: a meta-analysis of randomized clinical trials. Am J Med. 2006;119(12):1056-61.

6. Lagerqvist B, James SK, Stenestrand U, Lindbäck J, Nilsson $\mathrm{T}$, Wallentin L. Long-term outcomes with drug-eluting stents versus bare-metal stents in Sweden. N Engl J Med. 2007; 356(10):1009-19.

7. Eisenstein EL, Anstrom KJ, Kong DF, Shaw LK, Tuttle RH, Mark DB, et al. Clopidogrel use and long-term clinical outcomes after drug-eluting stent implantation. JAMA. 2007; 297(2):159-68.

8. Food and Drug Administration. Circulatory System Devices Advisory Panel Transcript for December 8, 2006, Meeting. Available at: http://www.fda.gov/ohrms/dockets/ac/06/transcripts/2006-4253t2.rtf. Accessed February 2, 2007.

9. Maisel $\mathrm{WH}$. Unanswered questions: drug-eluting stents and the risk of late thrombosis. N Engl J Med. 2007;356(10):981-4.

10. Farb A, Boam AB. Stent thrombosis redux - the FDA perspective. N Engl J Med. 2007;356(10):984-7.

11. Beohar N, Davidson CJ, Kip KE, Goodreau L, Vlachos HA, Meyers SN, et al. Outcomes and complications associated with off-label and untested use of drug-eluting stents. JAMA. 2007;297(18):1992-2000.

12. Win HK, Caldera AE, Maresh K, Lopez J, Rihal CS, Parikh MA, et al., EVENT Registry Investigators. Clinical outcomes and stent thrombosis following off-label use of drug-eluting stents. JAMA. 2007;297(18):2001-9.

13. Rao SV, Shaw RE, Brindis RG, Klein LW, Weintraub WS, Peterson ED. On- versus off-label use of drug-eluting coronary stents in clinical practice (report from the American College of Cardiology National Cardiovascular Data Registry [NCDR]). Am J Cardiol. 2006;97(10):1478-81.

14. Marroquin OC, Selzer F, Mulukutla SR, Williams DO, Vlachos HA, Wilensky RL, et al. A comparison of bare-metal and drug-eluting stents for off-label indications. N Engl J Med. 2008;358(4):342-52. 